\title{
A Novel Soundproof Ventilation Plant Design with High Performance and No Energy Consumption
}

\author{
Ya-Lin Yu, ${ }^{1,2}$ Yu-Tin Chao, ${ }^{2}$ Lai-Chung Lee, ${ }^{3}$ Jia-Yush Yen, ${ }^{2}$ and Yun-Chiu Fan ${ }^{4}$ \\ ${ }^{1}$ Graduate Institute of Design Doctoral Program, National Taipei University of Technology, Taipei 106, Taiwan \\ ${ }^{2}$ Department of Mechanical Engineering, National Taiwan University, Taipei 106, Taiwan \\ ${ }^{3}$ Department of Interaction Design, National Taipei University of Technology, Taipei 106, Taiwan \\ ${ }^{4}$ Department of Information System, The University of Texas at Dallas, TX 75080-3021, USA
}

Correspondence should be addressed to Lai-Chung Lee; f10666@ntut.edu.tw

Received 15 June 2014; Revised 16 October 2014; Accepted 28 October 2014

Academic Editor: Teen-Hang Meen

Copyright (C) 2015 Ya-Lin Yu et al. This is an open access article distributed under the Creative Commons Attribution License, which permits unrestricted use, distribution, and reproduction in any medium, provided the original work is properly cited.

\begin{abstract}
This paper proposes an advanced approach for designing soundproof windows with good ventilation. The structure of this study is based on three acoustics theorem and can reduce noise and ventilate the space at the same time. Using this structure on wall can help also avoid noise interference effect indoors and increase air quality. This study uses plants as appropriate soundproof structure to improve carbon dioxide content for providing better living environment. Simulations of the sound field and CFD demonstrate that the structure has good performance on noise reduction and effect of ventilation.
\end{abstract}

\section{Introduction}

Urbanization, high-density population, and traffic not only bring convenience but also cause noise pollution to us. The researches of [1-4] and others show that urbanization indeed caused noise pollutions, and those residents feel that their quality of life declines by noise environment.

Many researches pointed out that noise damages both our body and mind. In mental side, noise causes mental weakness, insomnia, headache $[5,6]$, anxiety, concentration difficulty, and so forth; in physical side, it leads to hearing loss $[7,8]$, immune system damage, and cardiovascular diseases $[9,10]$.

In addition, because the lifestyle and working style are changing constantly, now people spend over $80 \%$ of their time per day in a room. The standard for concentration of carbon dioxide is less than $1000 \mathrm{ppm}$. However, when the indoor carbon dioxide concentration exceeds $800 \mathrm{ppm}$ [11], people will get uncomfortable, tired, and headache.

This research includes three kinds of acoustic method. First, we discuss hush pipe principal which has the filtering utility. This principle is based on the difference of crosssection area along the path [12] and is always used in the condition which needs air circulation and noise reduction. Thus this kind of design is generally applied on vehicles [13]. Second, we discuss the double wall principal which is used to strengthen the noise reduction utility [14]. This design is always for indoor soundproof. The double wall design not only reduces the noise but also has the straight path of air flow. Unlike the traditional ventilating soundproof methods in which air flow needs detour [15] and is possible to reflect, the design in this study makes air flow into the house more easily.

In 2009 Yuya et al. proposed a design of soundproof ventilation window with good performance. This design is with detour flow route and the width of air vent is $17 \mathrm{~mm}$ [15]. In 2013, Kwon and Park proposed a design with big ventilation space; it uses two control sources based on feedforward control method for active noise control. The noise reduced by about $10 \mathrm{~dB}$ in the frequency band of 400 $1000 \mathrm{~Hz}$ by the proposed system [16]. In 2014, Wang et al. proposed a straight flow route with better noise reduction effect. But it is only working on limited frequency range and the effect is not good on low frequency. The thickness of this design is $800 \mathrm{~mm}$ and width flow route is $40 \mathrm{~mm}$; the design with $800 \mathrm{~mm}$ thickness is not feasible for practical use if it 
is set as window in normal family [17]. In our research we integrate all the problems in each design and propose a much more balanced design and have certain qualities in every aspect.

Overall, we provide much more equal noise reduction in different frequencies; the performance in low frequencies is also included. With regard to ventilation design, the area of flow route is also set as bigger size, and the design does not use any control source for noise reduction, so there is also no energy consumption. Considering the indoor environment, if there is no interferential noise, we can talk more clearly, so the third method is about how to reduce this kind of noise. Reference [18] proves that the rectangular anechoic chamber's sound absorption coefficient is more dispersed than the analytical data obtained by the pentagonal one, and [19] also proves this by the other experiment. By testing the same sample in different anechoic chamber, Katsu found that the results are different and the pentagonal one has the best result [20]. As a consequence, this research uses the regular pentagonal structure to construct the walls, doors, and windows.

Unlike the traditional soundproof equipment, in this research, we plant the tuber fern, daisy, or something that can absorb damaging substances in the soundproof equipment. The Boston fern is easy to plant, suitable to plant in windowsill and balcony, and good for reducing carbon dioxide, so this plant is preferred. Many researches have also proposed that green building can reduce pollution, absorb carbon dioxide and dust particles, and improve microclimate [21]. This research takes advantage of these points, constitutes the acoustic structure by natural plant, and allows the plant to grow along the shape of the structure.

This research combines all the points above and not only proposes the soundproof structure with ventilating utility but also uses green design to improve the conflict between deadening and ventilating. Moreover, this research can improve air quality and temperature regulation. Currently there is no such product like this, so this design is very innovative.

\section{Materials and Methods}

2.1. The Theory of the Traverse of a Pipe. In general, Muffler's theorem is always used on soundproofing design [22]. This is achieved by the different filtering effect to sounds of different wavelengths utilizing the relation between cross-sectional area and length.

Suppose that a sound wave comes from a pipe with the superficial area of cross-section $S_{1}$. At the extremity of $S_{1}$, it is penetrated with another pipe with the superficial area of cross-section, $S_{2}$. Consider the boundary conditions and assume that the sound field is not heterogeneous over the acoustic wavelength yielding the reflection coefficient and transmission coefficient of the sound intensity:

$$
t_{I}=\frac{I_{t}}{I_{i}}=\frac{4}{\left(S_{12}+1\right)^{2}}
$$

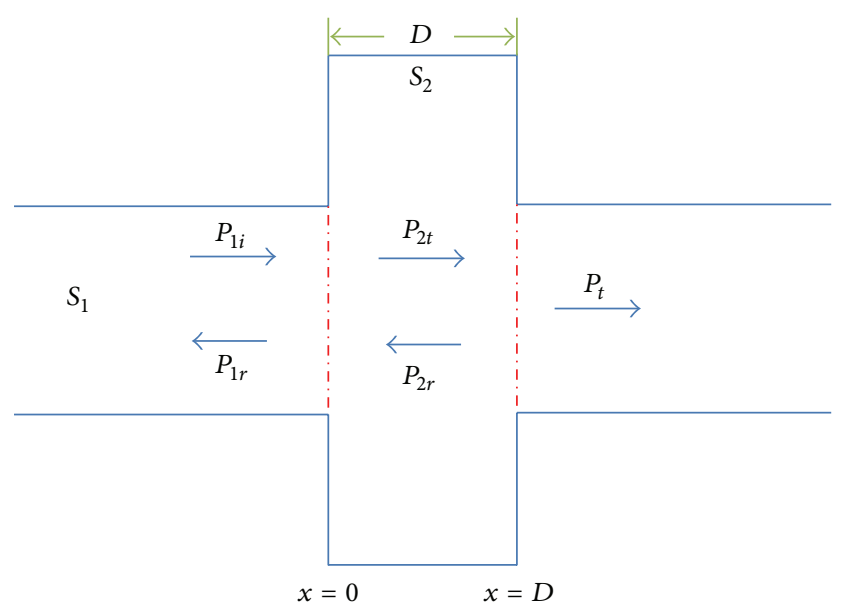

FIGURE 1: The traverse of expansion pipe.

where

$$
S_{12}=\frac{S_{2}}{S_{1}}, \quad S_{21}=\frac{S_{1}}{S_{2}},
$$

$S_{12}$ and $S_{21}$ are the ratio of the cross-sectional areas, and $t_{I}$ is transmission coefficient of sound intensity.

The average sound energy flow or power transmission coefficient can be expressed as

$$
t_{W}=\frac{I_{t} S_{2}}{I_{i} S_{1}}=\frac{4 S_{12}}{\left(1+S_{12}\right)^{2}} .
$$

Assuming a structure with the form shown in Figure 1, and according to the continuity conditions for the sound pressure and volume velocity, we can derive the following formulas for $x=0$ :

$$
\begin{gathered}
P_{1 i a}+P_{1 r a}=P_{2 t a}+P_{2 r a}, \\
S_{1}\left(v_{1 i}+v_{1 r}\right)=S_{1}\left(v_{2 t}+v_{2 r}\right),
\end{gathered}
$$

where $P_{1 i a}, P_{1 r a}, P_{2 t a}, P_{2 r a}$ are the amplitude of $P_{1 i}, P_{1 r}, P_{2 t}$, $P_{2 r}$.

When $x=D$,

$$
\begin{gathered}
P_{2 t a} e^{-j k_{2} D}+P_{2 r a} e^{j k_{2} D}=P_{t a}, \\
S_{2}\left(v_{2 t a} e^{-j k_{2} D}+v_{2 r a} e^{j k_{2} D}\right)=S_{1} v_{t a},
\end{gathered}
$$

where $P_{2 t a}, P_{2 r a}, P_{t a}, v_{2 t a}, v_{2 r a}, v_{t a}$ are the amplitude of $P_{2 t}, P_{2 r}$, $P_{t}, v_{2 t}, v_{2 r}, v_{t}$. 
The particle velocities are expressed as

$$
\begin{aligned}
& v_{1 i}=\frac{p_{1 i a}}{\rho_{0} c_{0}} e^{j(\omega t-k x)}, \\
& v_{1 r}=-\frac{p_{1 r a}}{\rho_{0} c_{0}} e^{j(\omega t+k x)}, \\
& v_{2 i}=\frac{p_{2 t a}}{\rho_{0} c_{0}} e^{j(\omega t-k x)}, \\
& v_{2 r}=-\frac{p_{2 r a}}{\rho_{0} c_{0}} e^{j(\omega t+k x)}, \\
& v_{i}=\frac{p_{t a}}{\rho_{0} c_{0}} e^{j(\omega t-k x)} .
\end{aligned}
$$

Substituting (6) into (4) and (5) yielding the ratio of sound pressure of the transmitted wave at $x=D$ to the incident wave at $x=0$ (after some algebraic operations),

$$
t_{p}=\frac{p_{t a}}{p_{i a}}=\frac{2}{\left[4 \cos ^{2} k_{2} D+\left(S_{12}+S_{21}\right)^{2} \sin ^{2} k_{2} D\right]^{1 / 2}}
$$

The ratio of the intensities of the transmitted and incident waves is called the sound intensity transmission coefficient, which can be expressed as

$$
\begin{aligned}
t_{I} & =\frac{I_{t}}{I_{i}} \\
& =\frac{\left|P_{t a}\right|^{2} / 2 \rho_{0} c_{0}}{\left|P_{i a}\right|^{2} / 2 \rho_{0} c_{0}}=\frac{4}{4 \cos ^{2} k_{2} D+\left(S_{12}+S_{21}\right)^{2} \sin ^{2} k_{2} D} .
\end{aligned}
$$

By referring to (6), it is evident that sound waves after the transmissions of various pipes with one's own superficial area of cross-section not only have the correlation with the ratio of one's own superficial area of cross-section but also have the lengths of those pipes. Ordinary noises around the vicinity of common families are mainly medium low frequencies. For example, the frequency of noise of an engine is about $125 \mathrm{~Hz} \sim 1000 \mathrm{~Hz}$, and the one caused by common people is about $100 \mathrm{~Hz} 2000 \mathrm{~Hz}$. According to the theory of the traverse of a pipe, the velocity of sound is about $343 \mathrm{~m} / \mathrm{per}$ second. This research proposes the structure matched for $686 \mathrm{~Hz}$. When using a pipe with $12.5 \mathrm{~cm}$ traverse superficial area, it has the weakest capability of penetration as in Figure 2. However, it is found that a pipe with $12.5 \mathrm{~cm}$ traverse superficial area has the best ability of penetration as in Figure 3. Under this frequency, noises are not lowered. In order to ameliorate this problem, this research is devoted to the feeblest perforating capability of $1372 \mathrm{~Hz}$ as in Figure 4. A pipe with $6.25 \mathrm{~cm}$ superficial area of cross-section can isolate the noise of $1372 \mathrm{~Hz}$ first rate. Generally, outdoor noises are under $2000 \mathrm{~Hz}$; the combination of $12.5 \mathrm{~cm}$ and $6.25 \mathrm{~cm}$ is sufficient for the separation of undesired sound, as shown in

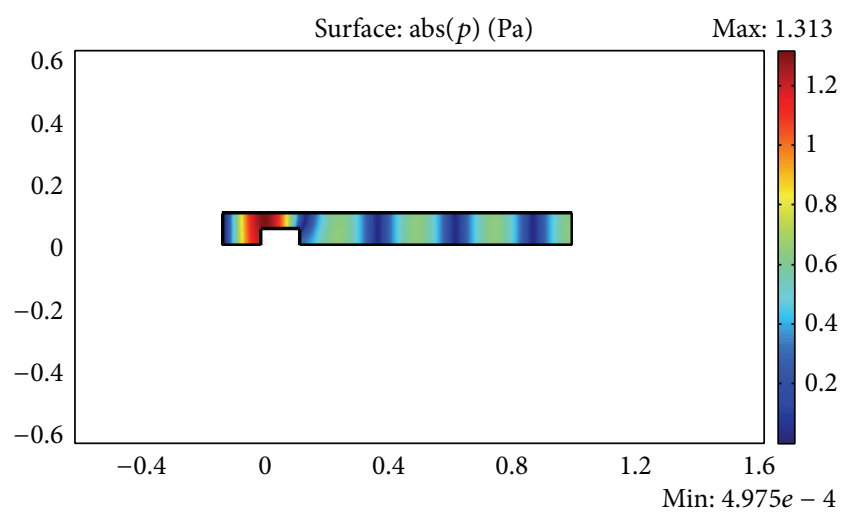

FIgURE 2: A pipe with $12.5 \mathrm{~cm}$ traverse superficial area in height (excited frequency: $686 \mathrm{~Hz}$ ).

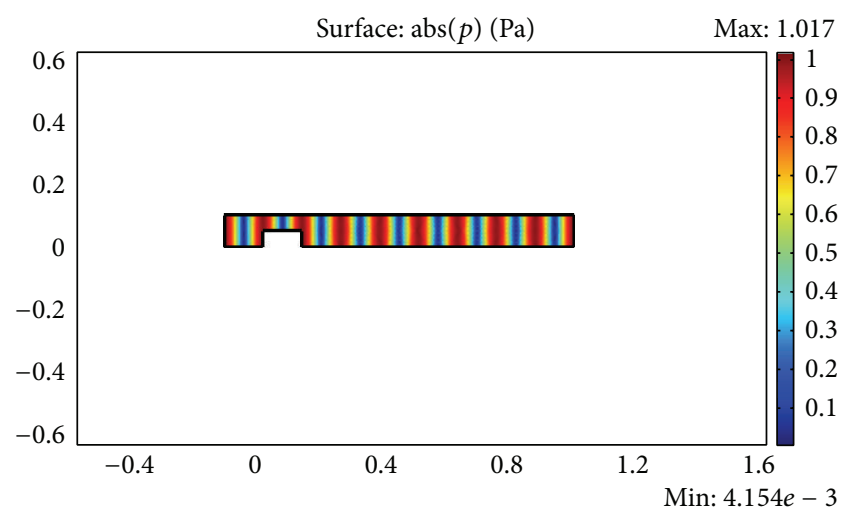

Figure 3: A pipe with $12.5 \mathrm{~cm}$ traverse superficial area in height (excited frequency: $1372 \mathrm{~Hz}$ ).

Figure 5. Consequently, our focus is the fuse of $12.5 \mathrm{~cm}$ and $6.25 \mathrm{~cm}$ pipes. The result of simulation matches our theory. This design is capable of declining outdoor rumble from hundreds to $2000 \mathrm{~Hz}$, even though, in high frequency, the noise is still lowered down. This invention can plunge noises and simultaneously offer ample space for the circulation of air.

2.2. The Theory of Lateral Pentagon Design. Because of the space effect, the complex sonic received by human's ears is divided into direct sound, early reflection, and later reflection [23], as in Figure 6.

Sound waves going directly into the listener's ear without reflection are the direct sound. Listeners can learn the amplitude of sound sources, sound quality, and so forth from the direct sound.

Early reflected sound is the sound waves that bump into walls, ceilings, floors, and desktop and then do a reflective sound and finally go into human's ears. The time difference compared to the direct sound is about 10/1000-30/1000 seconds. If the reflection path of sound is too longer and makes the residual sound more than 50/1000 seconds (1/20 seconds), it will generate an obvious interval with direct sound. This sounds like two consecutive voices called echo. 


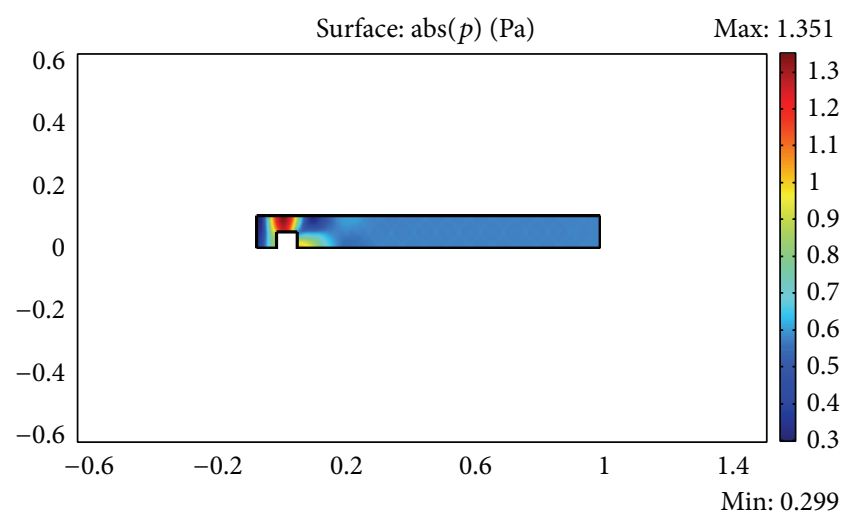

Figure 4: A pipe with $6.5 \mathrm{~cm}$ traverse superficial area in height (excited frequency: $1372 \mathrm{~Hz}$ ).

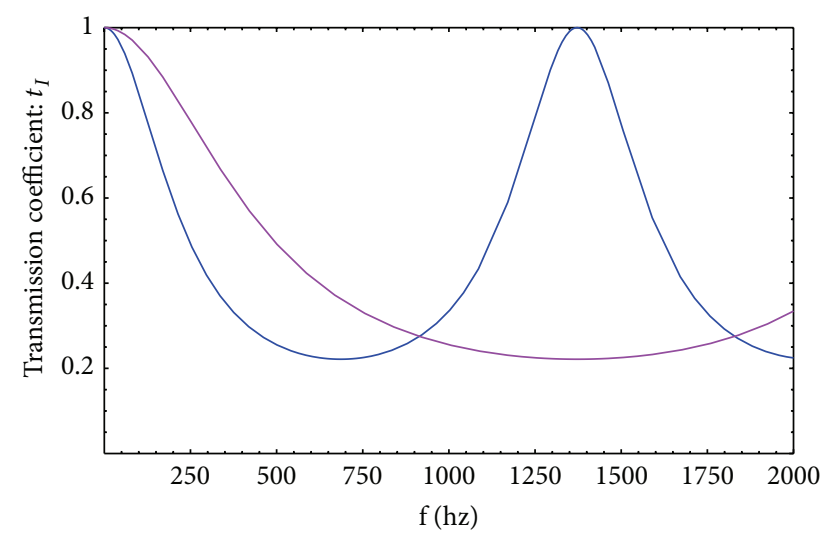

FIgURE 5: The transmission coefficient of expansion tubes. Blue line $(D=12.5 \mathrm{~cm})$ and purple line $(D=6.25 \mathrm{~cm})$.

If the space is too small so that these sound waves overlap each other, it is called flutter echo. Because residual sound or echo would reduce the clarity of the sound, sound generated interference by the phase difference, waves overlapping in space form a new waveform, and then the quality of sound declines. It is the problem that this research wants to ameliorate.

This study compared the structural design with different boundary, discussing their situation of reflection and the influence of interference, as in Figures 7, 8, and 9. Under the situation of incident and reflective surfaces paralleling with each other, incident sound source directly generates reflected sound and interferes with original sound. Under this case, the reflective sound source is affected one time by the impedance of the reflective surface, so the amplitude of sound pressure is still big. With this situation, incident sound source is affected more by reflection interference. If the design of the angle between the incident source and the reflecting surface is 45 degrees, the sound source will generate opposite reflection after two reflections. Its reflected sound is influenced by impedance of material surface by two times and the center line of the reflection path and

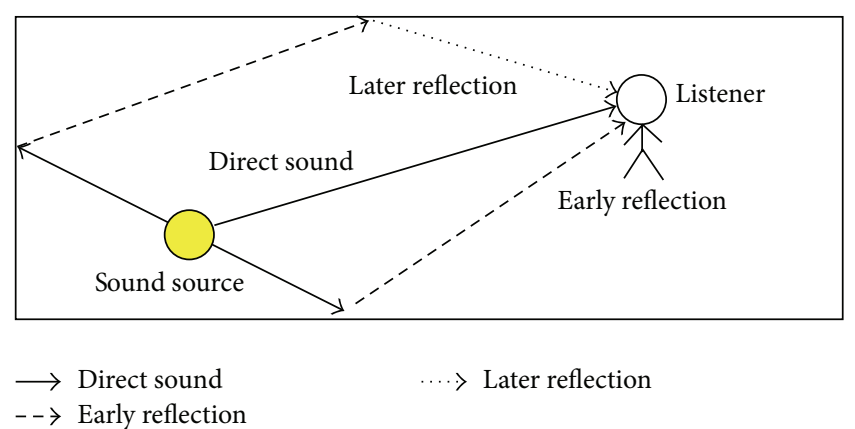

FIGURE 6: The diagram of acoustic path of confined space.

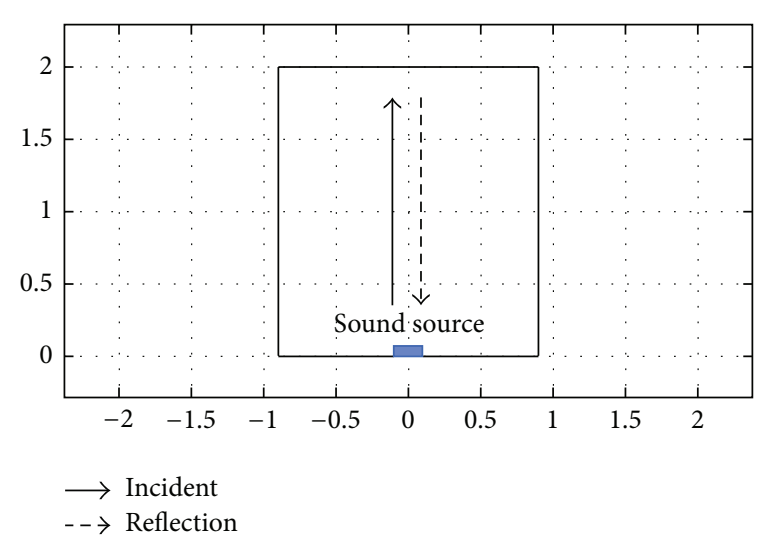

FIgURE 7: The sound reflection figure of incident sound source radiating to a wall without angle.

the center line of the incident path have a certain distance, so that the sound pressure of the reflected wave of the structural design of 45 degrees is smaller than incident and reflective surfaces paralleling with each other. However, if we use a pentagonal design with an included angle, 54 degrees, between incident source and reflecting surface, under this design with two reflections, reflected sound is less affected by the characteristic impedance of the two materials than amplitude. Otherwise, the center line of the reflection path and the center line of incident path have a certain distance. So the sound pressure of the structural design of 45 degrees is smaller than the incident and reflective surfaces paralleling with each other. However, when using a pentagonal design with an included angle, 54 degrees, between incident source and reflection surface, under this design with two reflections, the amplitude of reflected sound is smaller because the reflected sound is influenced by impedance of material surface by two times and the reflected sound is more away from the sound source when the path is longer. So as long as a certain distance is set from the sound source to structure, the sound is more alike the original sound. Compared to other structures, pentagonal angle design decreases interference problems. If the angle of the side structure is designed as a pentagon, it will be able to effectively improve the interference problems. 


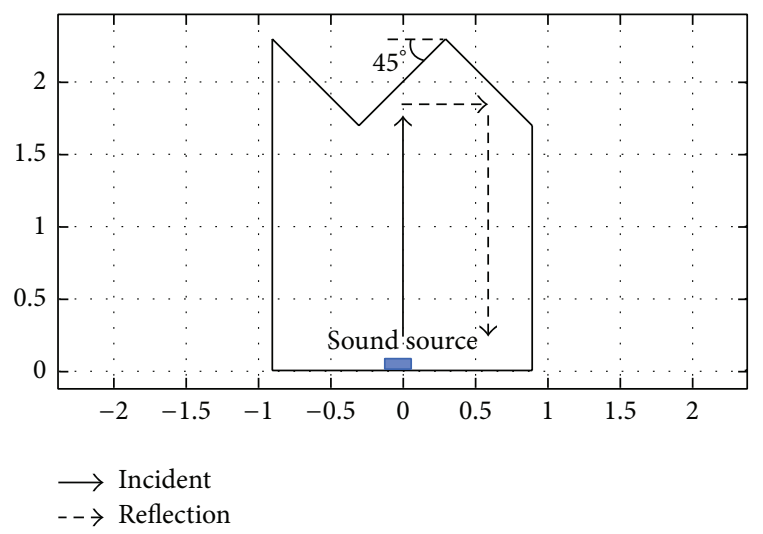

FIgURE 8: The sound reflection figure of incident sound source radiating to a wall with 45 -degree angle.

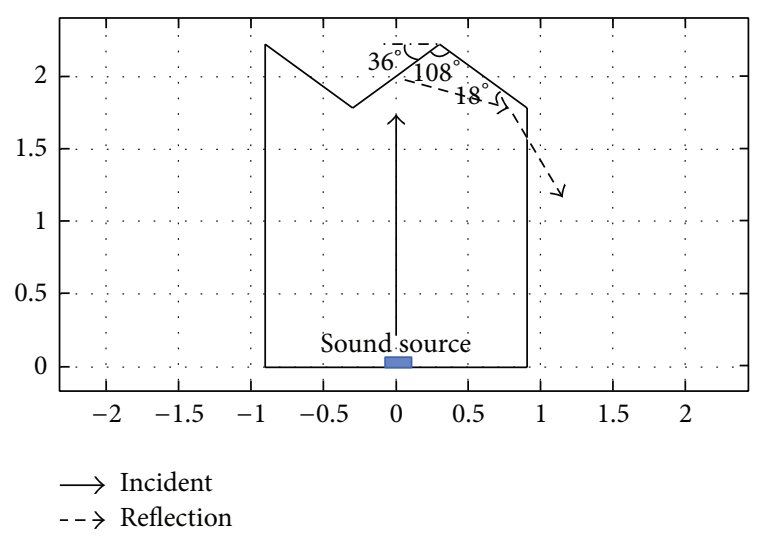

FIGURE 9: The sound reflection figure of incident sound source radiating to a wall with 108-degree angle.

2.3. Theory of Double-Layer Wall. Generally, it is expected that a residence with walls, doors, and windows has excellent isolation from noises. The best part of the structures of walls and doors is a solid body. The property of a solid structure has much greater isolating ability than air and most design of walls is multilayer. Therefore, when consuming equal amount of building materials, a design with a more efficacious ability to separation from noises is presented. When the conditions of a multilayer wall $\omega M / 2 R \gg 1$ are reached ( $M$ is mass per unit region; $R$ is the characteristic impedance), the separating ability, filtering noises, of a one-layer wall is shown as $T L=$ $10 \log _{10}(\omega M / 2 R)^{2}[22]$.

In conclusion, it is comparatively effortless for a doublelayer wall to isolate sound, besides under low frequency. A wall separated into 2 layers is beneficial to obstruct sounds. Our research takes advantage of double-layer structure and the theory of the traverse of a pipe. Otherwise, we integrate the pentagon design of isolating mechanism, not easy to generate interference noise, and utilize the traverse of a pipe, offering sufficient space for the circulation of air. Finally, the structure is capable of descending sound with excellent circulation of air being reached.

\section{Results and Discussion}

3.1. The Arrangement of Space of the Devised Structure. However, the route mentioned above is designed to integrate anti-interference of the pentagon. When the size of the traverse of a pipe is made sure in the second section, the middle part of the path $(12.5 \mathrm{~cm}$ and $6.25 \mathrm{~cm}$ traverse pipes) is aimed for establishing natural structure, growing plants (as shown in number 2 red sign of Figure 10). This design is to let the shape of plant structure alike the convex structure in Figures 2 and 4 and can also provide better air quality at the same time. A double-layer wall is also carried to place the two compensate structures side by side for a better effect of isolating sounds. The entire approaches of ventilation and closure are shown in Figure 11. The two different thicknesses are the best and the worst capability of penetration, referred to in chapter 2, coming from the outcome of cross design. This design offers a better effect of isolation of sounds.

3.2. Double-Wall Structure Simulation. According to the space arrangement mentioned above, we can define the lateral plane propagated method in Figure 12, so this research simulates the route as in Figure 13. Considering the indoor space and walls for the path, the combination of $12.5 \mathrm{~cm}$ and $6.25 \mathrm{~cm}$ pipe is our preference and has another merit, the effect of a double-layer wall. In order to eliminate noise sound and ventilate the space well by the organization of the space, sound field and wind flow are probed, respectively. When it comes to the analysis of sound field, we focus on the incidence condition under $100 \mathrm{~Hz}, 1000 \mathrm{~Hz}$, and $2000 \mathrm{~Hz}$. The setting up of condition, the whole boundary condition, is shown in Figure 13, where black broken lines mean matching boundary and black real lines mean sound hard boundary. This research can block low frequency of outdoor noises as shown in Figures 14, 15, and 16. Compared with initial space arrangement with and without the soundproof designed structure, we yield the transmission loss on measured point as shown in Figure 17, where without the designed structure 5 is remove the red broken line region from Figure 13, as a plane wall structure. This result shows that the transmission loss is over $10 \mathrm{~dB}$ when the frequency is only $300 \mathrm{~Hz}$, and the soundproof effect is over $10 \mathrm{~dB}$ when the frequency is $300-$ $2000 \mathrm{~Hz}$.

In terms of the analysis of wind flow, we also have the same arrangement of space like Figure 18. This design includes wind flow with one meter per second. It is found that when the experiment starts $(t=0)$, wind flow goes in all directions. Consequentially $(t=1)$, streamline moves to the right-low direction because of the influence of wind and structure. In accordance with the outcome of Figures 1920 , it shows that the air circulates indoors. The total width of air vents in this study is $150 \mathrm{~mm}$ and it is bigger than most of relative designs. Integrating all the mentioned above, the design combining the $12.5 \mathrm{~cm}$ and $6.25 \mathrm{~cm}$ traverse pipes can eliminate noises and ventilate the space well simultaneously.

3.3. The Spatial Sound Field Simulation of Lateral Structure Design. Different interference of conditions is generated 

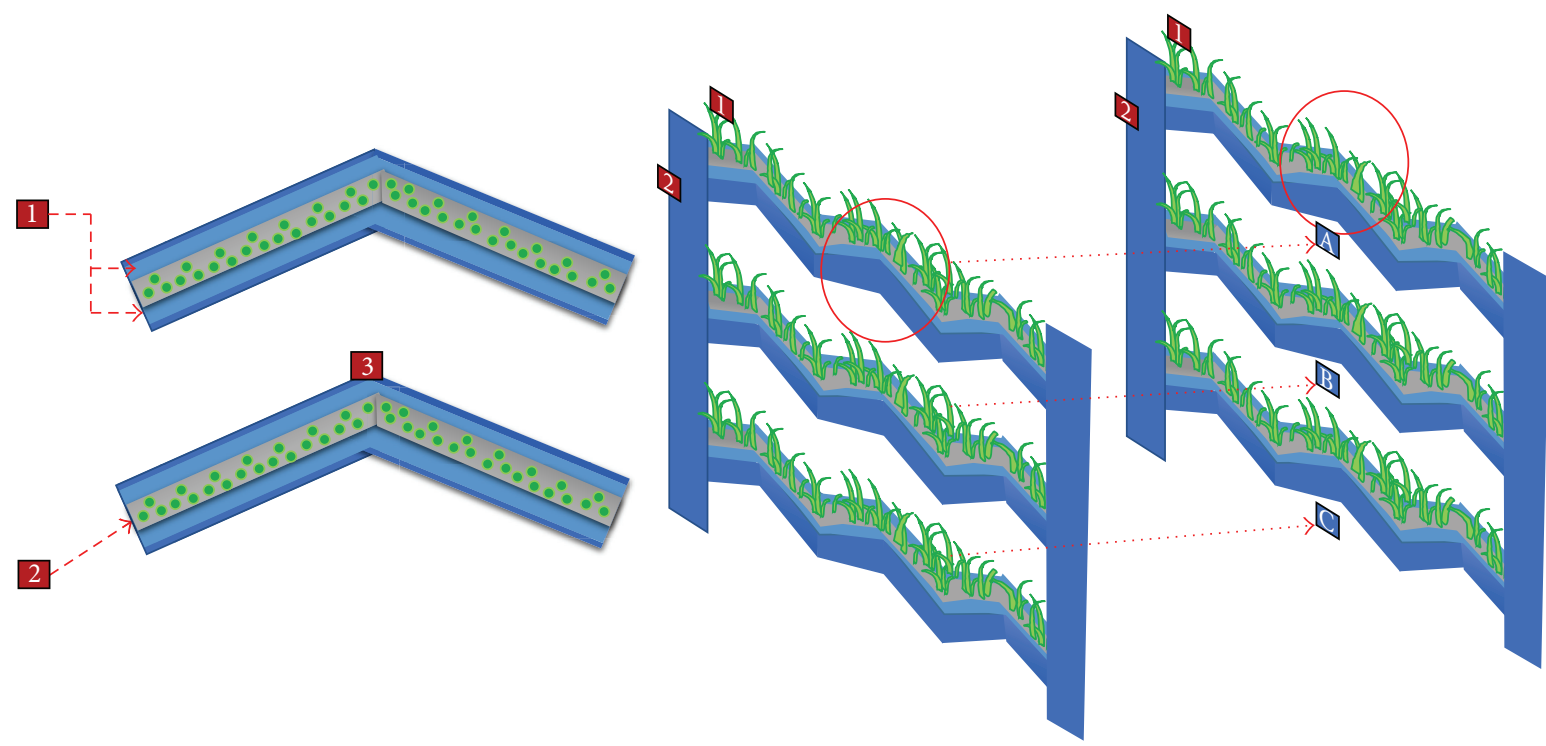

Double wall

Plant

FIGURE 10: The systematic diagram of the structure of isolating sound and circulating air.
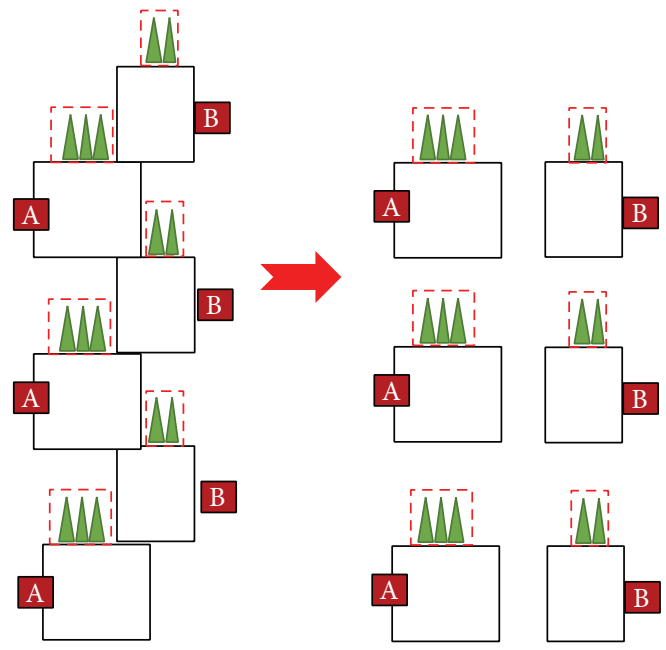

FIGURE 11: The sectional drawing of the structure of isolating sound and circulating air.

by different lateral structures; the basic theory has been proposed (see Section 2.2). And the design of pentagonal included angle of 108 degrees is proposed less likely to be affected by interference than the data of other test included angles. We simulate different lateral shape and the arrangement of boundary conditions is set as in Figure 21. From the results, we propose that pentagon structure is indeed similar to the original signal compared to the design of plane or included angle of 45 degrees.

For an indoor sound field, we set a sound source with a radius of $10 \mathrm{~cm} 1000 \mathrm{~Hz}$, placed in a wall of indoor space. Assuming the design of the lateral-shape border is on the

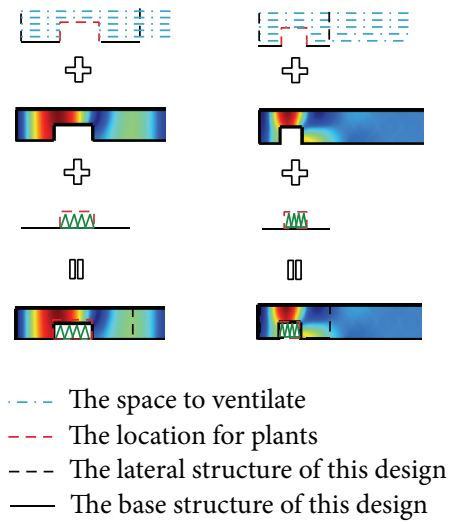

FIgURE 12: The arrangement of the two muffler structures and ventilation of spaces.

opposite side of the wall with the distance of $2 \mathrm{~m}$ from it, the material is glass, where density is $1150 \mathrm{~kg} / \mathrm{m}^{3}$ and sound speed is $5300 \mathrm{~m} / \mathrm{s}$. For lateral structure, observe how the sound of straight line sound field is influenced by interference.

We can learn from Figure 22, assumes that the sound pressure of original sound source is as the solid line and propagate following the route which shows in Figures 7-9, where the lateral structure of the models is designed as a plane, a 45-degree included angle and a 108-degree included angle. Compare with three lateral structure, the interference influence is different because the natural reflection route is different. The simulation results show that the sound pressure near the close structural design is more oppressive. Part of the data structure near the glass is still affected by the reflected-sound interference, but when being away from the 


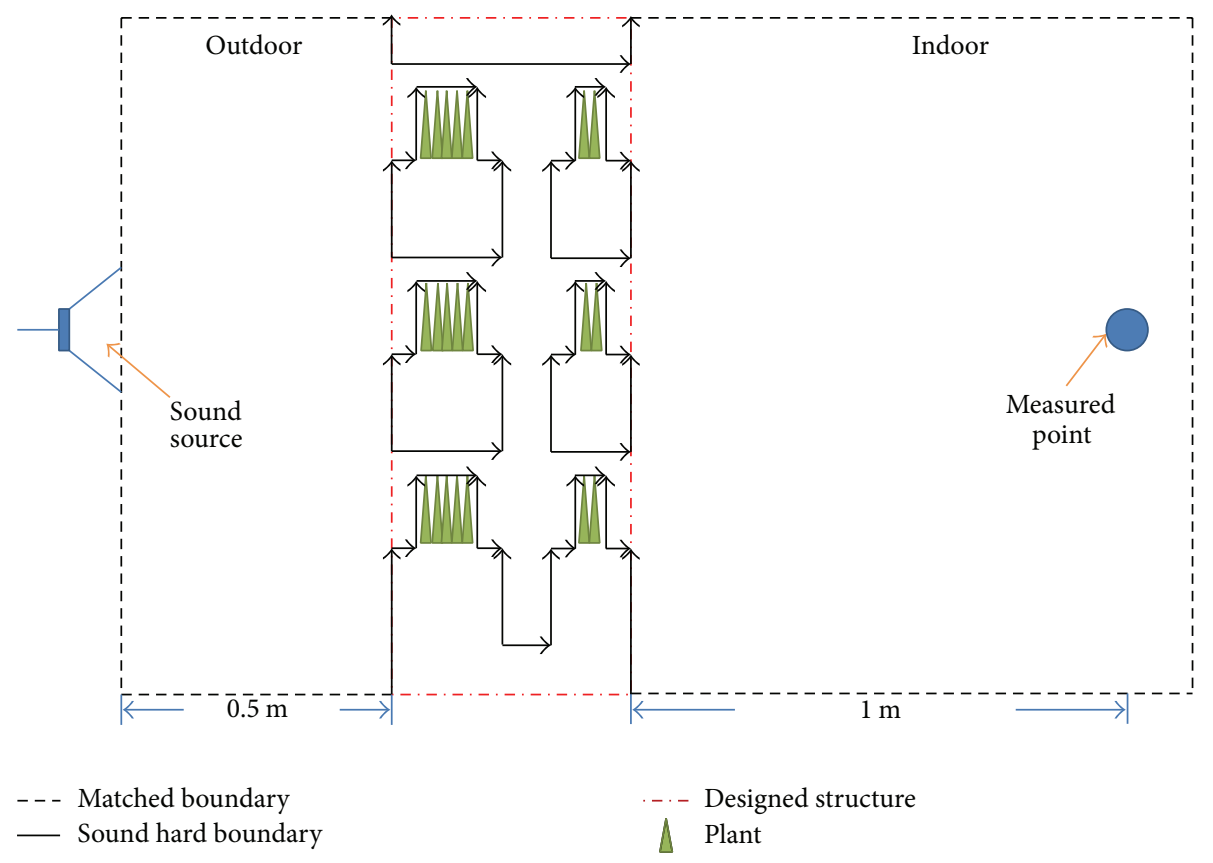

FIgURE 13: The design for the structure of reducing noises.

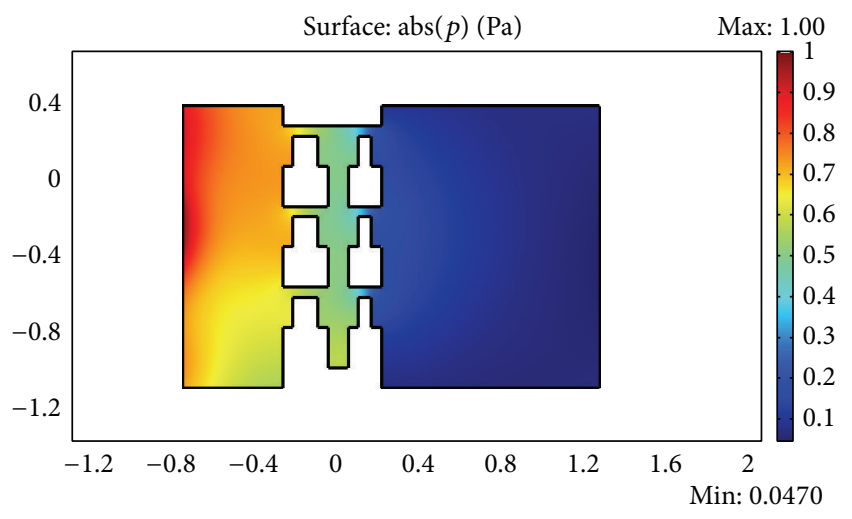

FIGURE 14: The sound pressure distribution of the indoor space under $100 \mathrm{~Hz}$.

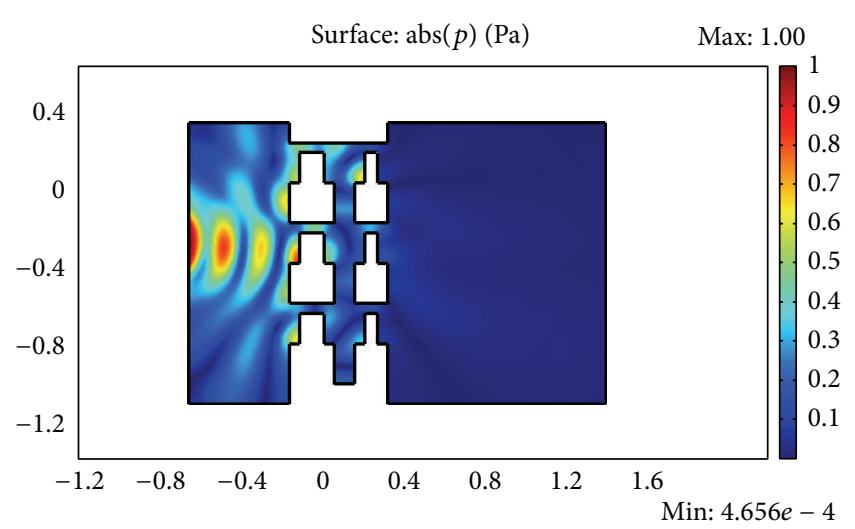

FIGURE 15: The sound pressure distribution of the indoor space under $1000 \mathrm{~Hz}$.

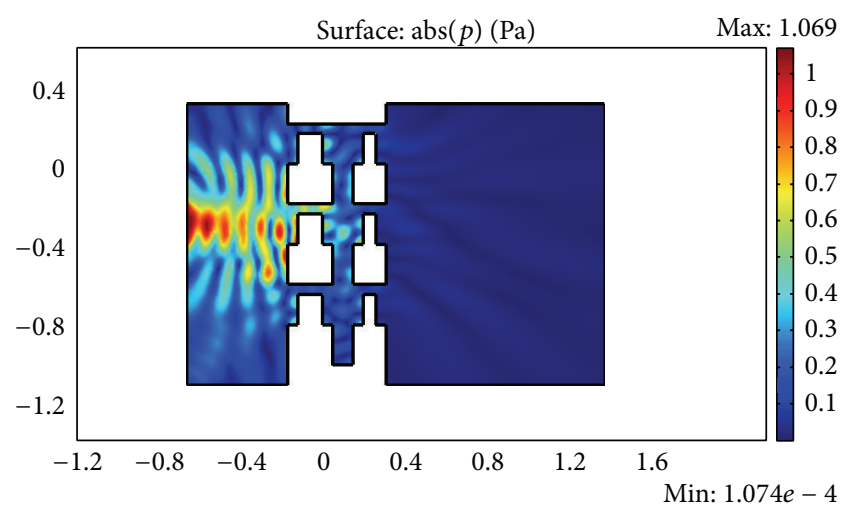

FIGURE 16: The sound pressure distribution of the indoor space under $2000 \mathrm{~Hz}$.

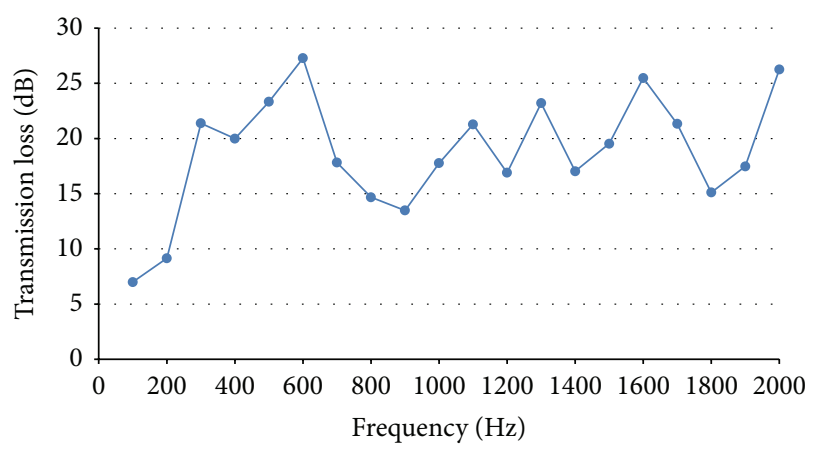

Figure 17: Transmission loss on measured point.

design of the structure or near the field of the sound source, pentagonal shape structure is apparently closer to the original 


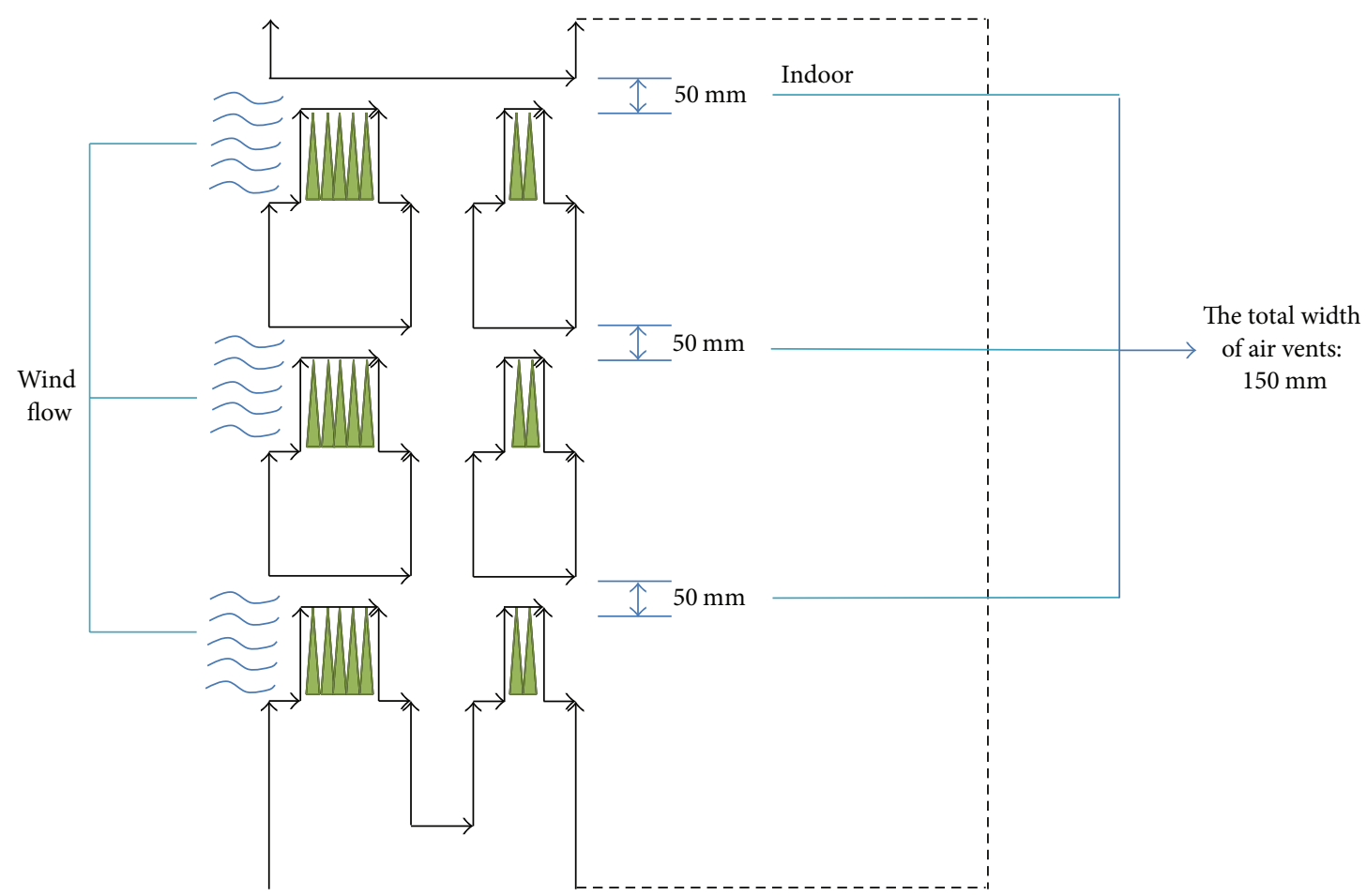

- Matched boundary
Sound hard boundary
Plant

FIGURE 18: The design for the structure of circulating air.

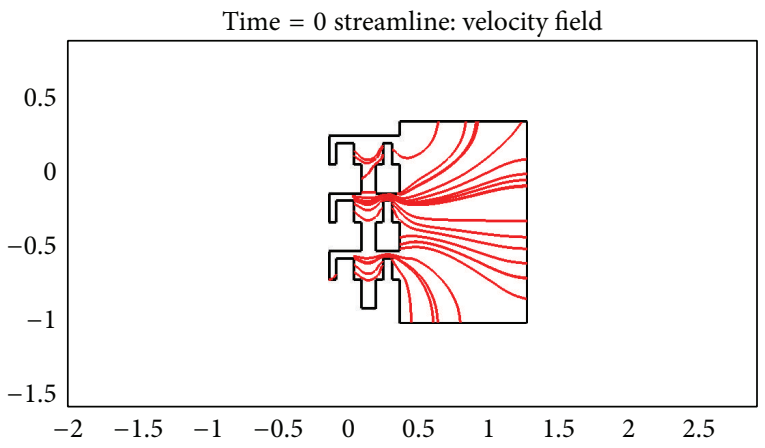

FIgURE 19: When $t$ is 0 , the velocity of air is shown above. Streamlines (30).

signal than other shape data. Because plane and 45-degree styles are affected, respectively, by one-time and two-time reflection sound, overall data has a big difference when compared with the original sound source. Plane style has the biggest difference owing to the influence of direct one-time reflection sound. Simulation results from the above show that if the soundproof-ventilation structure is designed as an included angle, it can indeed make indoor sound quality improve, let the received signal more similar to the original signal. especially when the lateral structure is designed with a 108-degree included angle.

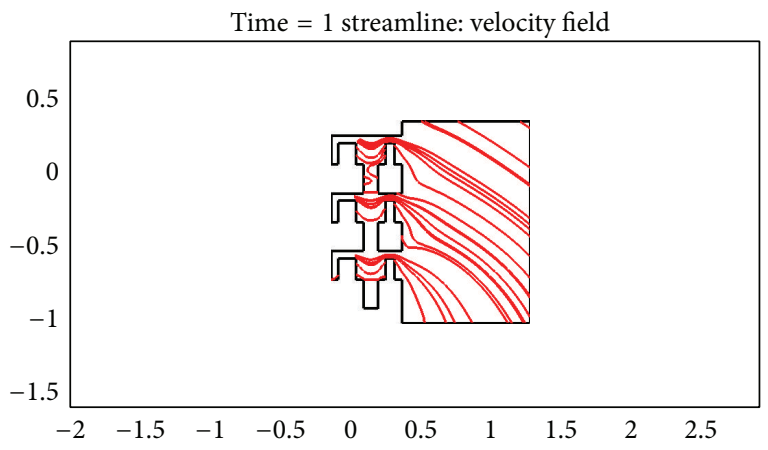

FIGURE 20: When $t$ is 1 second, the velocity of air is shown above. Streamlines (30).

Output through the results mentioned above shows that combining three theorems can improve sound quality on two propositions. The first proposition is to reduce outdoor noise; this kind of sound is propagated from outdoor source. Muffler's theorem is used to reduce a specific range of frequencies of noise, by using two muffler structures to set as double wall can give a bigger frequency range of soundproof. In this paper we design two muffler structures which compensate each other in the soundproof effect of different frequency range and provide better sound performance under $2000 \mathrm{~Hz}$ (Figure 17). The second proposition is to reduce the interference of reflected-sound indoor because this kind of sound is 


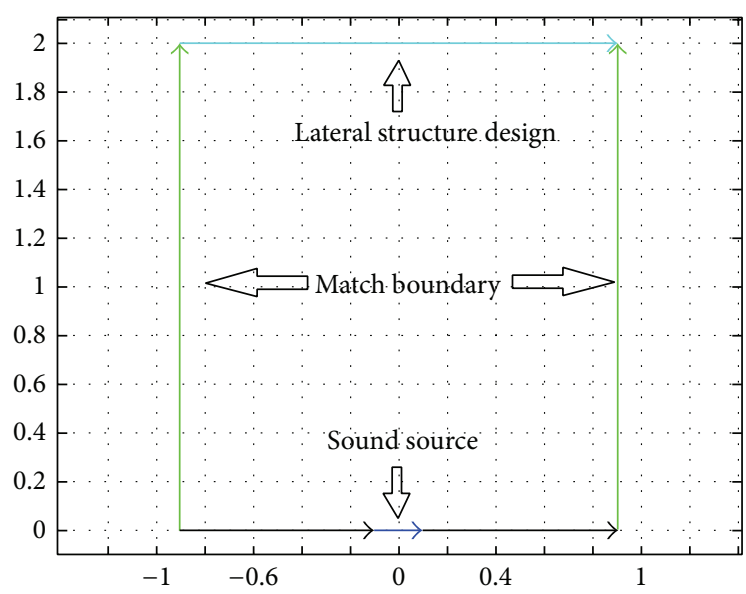

FIGURE 21: The boundary conditions setting for simulation.

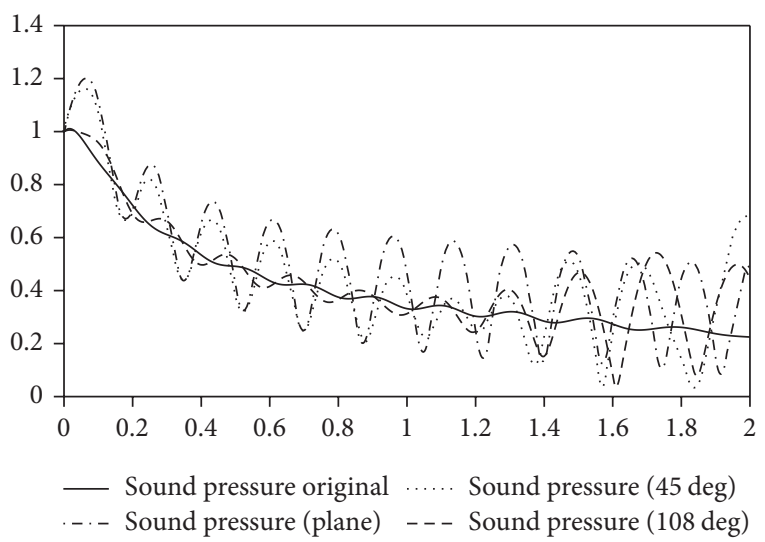

Figure 22: The sound performance of different structural design which is influenced by the reflection of the sound source under $2 \mathrm{~m}$ straight line lateral space.

made by indoor source, different from the initial condition of first research, so this part will be discussed separately. After simulation, we received signal more similar to the original signal through the lateral structures design with a 108-degree included angle, so we set our structure with 108degree included angle (Figure 10). The design in this paper improves sound quality and considers not only outdoor noise influence but also indoor interference; compared with relative researches, they seldom consider indoor interference at the same time, so this design is a novel design.

\section{Conclusion}

This research presents a brand new method of design style of structure with the capability of isolating noises and circulating well. We integrate three different theories of the physics of sound. Those theories are the traverse of a pipe derived from the principle of injection and reflection, doublelayer wall reducing noises, and the pentagon design for anti-interference. Generally, the model of sound physics and math is concordant with the simulation. The structure for diminishing noises performs well in sound field with low frequencies and the stimulation also corroborates its great effect of air circulation under the same conditions.

Compared with recent relative researches, they give less space for ventilation $[15,17]$, and the structure does not include compensated design, so the sound performance after noise reduction in some frequencies is of inferior quality, especially in low frequency $[16,17]$. In our research because of the compensated design, we provide better soundproof effect in low frequency and the soundproof effect performs much more equally under different levels of $2000 \mathrm{~Hz}$. The soundproof effect is over $10 \mathrm{~dB}$ from $300-2000 \mathrm{~Hz}$ and it is better than many relative designs. Our design does not use control sources for noise reduction, but some of the researches do use control systems for reducing noise; it needs continuous energy consumption and the control effect is still not obvious [16]. What size the window is is also considered in this design; some of designs are too big for being set in house [17], and the lateral structure design is also seldom under consideration [15-17]. For better listening environment with less interference, we also design the lateral structures with an included angle. Taking ventilation aspects of this study into account, we use double wall with straight flow route; the width for ventilation is $150 \mathrm{~mm}$ and there is no limit at length required. The design of ventilation condition is better than most of relative designs $[15,17]$.

In terms of purifying air, the plant style of sound field structure, innovative design, arranges plants into a natural structure to ease the influences of noises and furthermore resolves the problem of the excess of indoor carbon dioxide. In short, the unique design reaches our purpose of air circulation, the isolation from noises, the anti-interference of indoor noises, and the purifying of the environment.

\section{Conflict of Interests}

The authors declare that there is no conflict of interests regarding the publication of this paper.

\section{References}

[1] P. H. T. Zannin, F. B. Diniz, and W. A. Barbosa, "Environmental noise pollution in the city of Curitiba, Brazil," Applied Acoustics, vol. 63, no. 4, pp. 351-358, 2002.

[2] M. A. Sattler, "Urban noise survey for the city of Porto Alegre," Acustica, vol. 85, article 356, 1999.

[3] T. Kihlman, "City traffic noise-a local or global problem," 1999.

[4] S. H. Jhih, "The Study of Urban traffic noise prediction model with the reaction of residents," 1981.

[5] J. R. Goldsmith and E. Jonsson, "Health effects of community noise," American Journal of Public Health, vol. 63, no. 9, pp. 782793, 1973.

[6] S. Melamed and S. Bruhis, "The effects of chronic industrial noise exposure on urinary cortisol, fatigue, and irritability: a controlled field experiment," Journal of Occupational \& Environmental Medicine, vol. 38, no. 3, pp. 252-256, 1996.

[7] T.-J. Chen and S.-S. Chen, "Effects of aircraft noise on hearing and auditory pathway function of school-age children," International Archives of Occupational and Environmental Health, vol. 65, no. 2, pp. 107-111, 1993. 
[8] W. Passchier-Vermeer and W. F. Passchier, "Noise exposure and public health," Environmental Health Perspectives, vol. 108, no. 1, pp. 123-131, 2000.

[9] S. A. Stansfeld and M. P. Matheson, "Noise pollution: nonauditory effects on health," British Medical Bulletin, vol. 68, pp. 243-257, 2003.

[10] V. Regecova and E. Kellerova, "Effects of urban noise pollution on blood pressure and heart rate in preschool children," Journal of Hypertension, vol. 13, no. 4, pp. 405-412, 1995.

[11] O. A. Seppänen, W. J. Fisk, and M. J. Mendell, "Association of ventilation rates and $\mathrm{CO}_{2}$ concentrations with health and other responses in commercial and institutional buildings," Indoor Air, vol. 9, no. 4, pp. 226-252, 1999.

[12] L.-J. Yeh, Y.-C. Chang, M.-C. Chiu, and G.-J. Lai, "GA optimization on multi-segments muffler under space constraints," Applied Acoustics, vol. 65, no. 5, pp. 521-543, 2004.

[13] D. Potente, "General design principles for an automotive muffler," in Proceedings of Acoustics, 2005.

[14] C. Bao and J. Pan, "Active acoustic control of noise transmission through double walls: effect of mechanical paths," Journal of Sound and Vibration, vol. 215, no. 2, pp. 395-398, 1998.

[15] N. Yuya, N. Sohei, N. Tsuyoshi, and Y. Takashi, "Sound propagation in soundproofing casement windows," Applied Acoustics, vol. 70, no. 9, pp. 1160-1167, 2009.

[16] B. Kwon and Y. Park, "Interior noise control with an active window system," Applied Acoustics, vol. 74, no. 5, pp. 647-652, 2013.

[17] Z. H. Wang, C. K. Hui, and C. F. Ng, "The acoustic performance of ventilated window with quarter-wave resonators and membrane absorber," Applied Acoustics, vol. 78, pp. 1-6, 2014.

[18] K. Katsu, "The influence from the coefficient of sound absorption to a reverberation chamber," Acoustical Society of Japan, vol. 13, no. $4,1957$.

[19] S. Sakamoto, "The influence from measuring sample to area in a reverberation room," Acoustical Society of Japan, vol. 7, 2003.

[20] K. Katsu, "A study of the method to test the accuracy of sound absorption coefficient in a reverberation chamber," Acoustical Society of Japan, vol. 24, no. 6, 1968.

[21] S. Abbaszadeh, L. Zagreus, and D. Lehrer, "Occupant satisfaction with indoor environmental quality in green buildings," Proceedings of Healthy Building, vol. 3, pp. 365-370, 2006.

[22] E. Kinsler, A. R. Frey, A. B. Coppens, and J. V. Sanders, Fundamentals of Acoustics, 1999.

[23] J. C. Hou, Acoustics and Multimedia Audio Technology, Taiwan Commercial Press, 2007. 


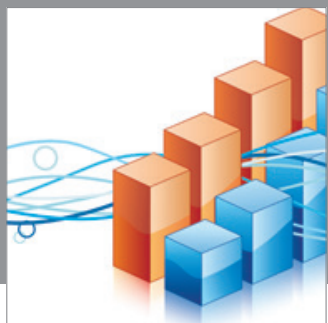

Advances in

Operations Research

mansans

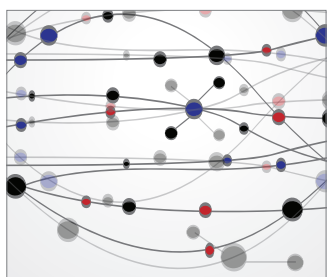

The Scientific World Journal
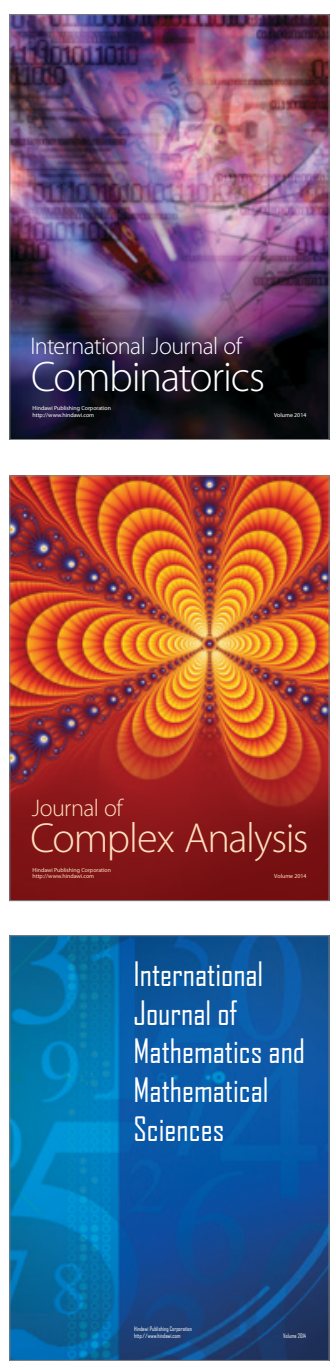
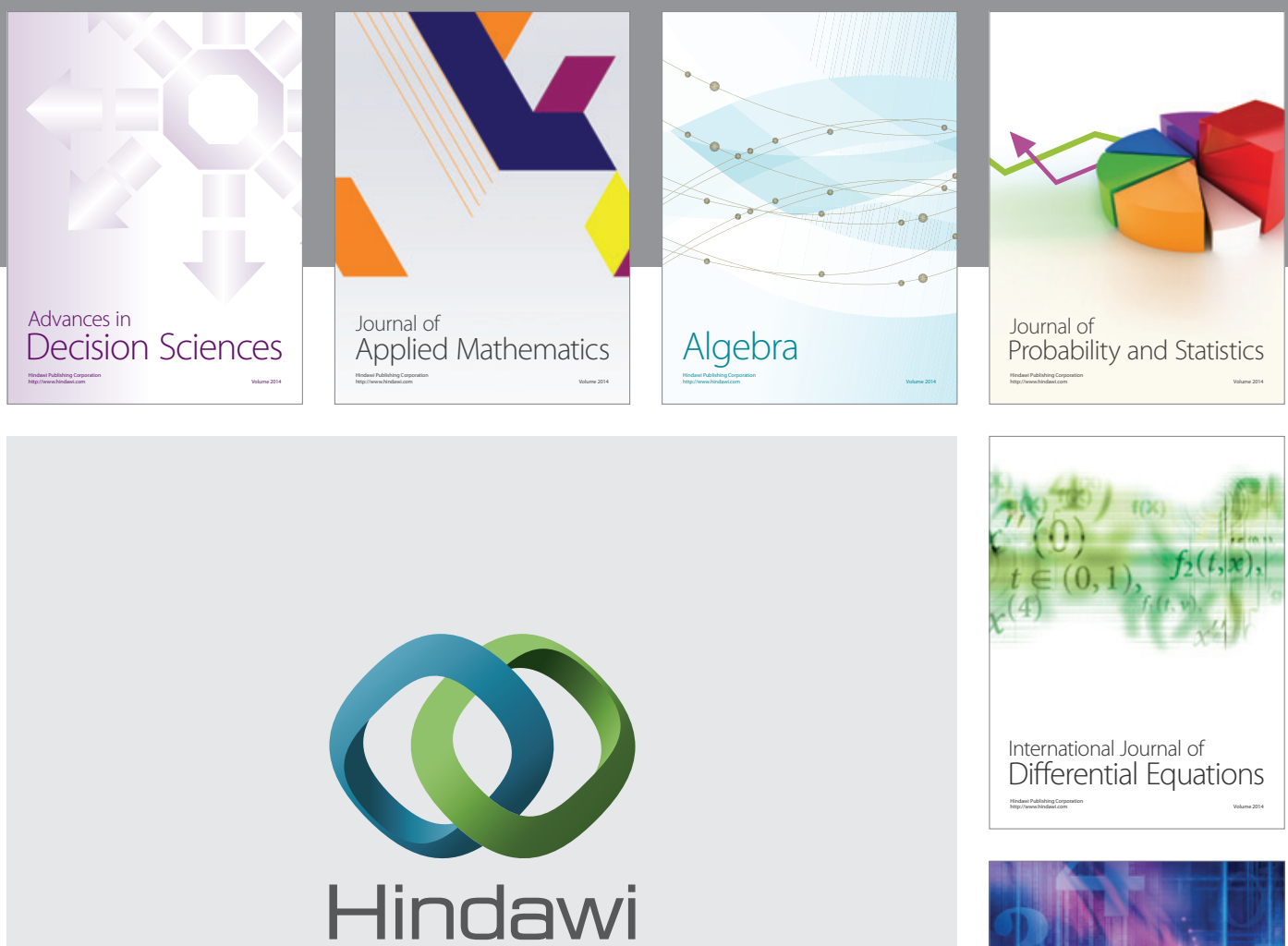

Submit your manuscripts at http://www.hindawi.com
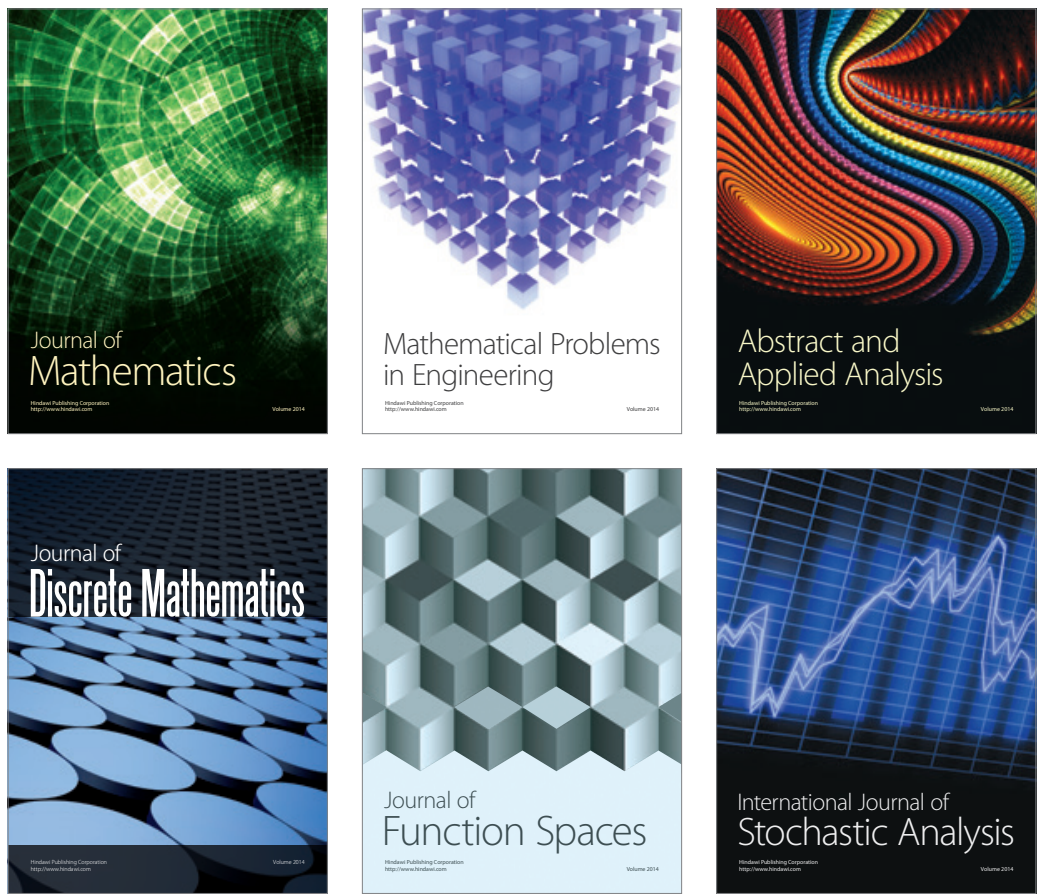

Journal of

Function Spaces

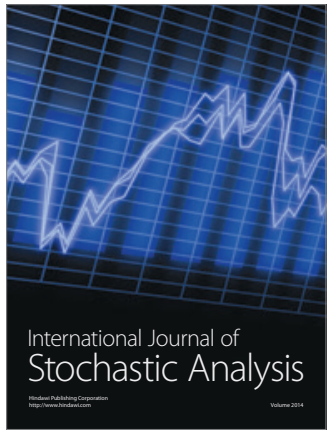

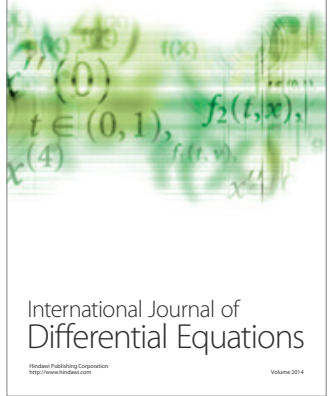
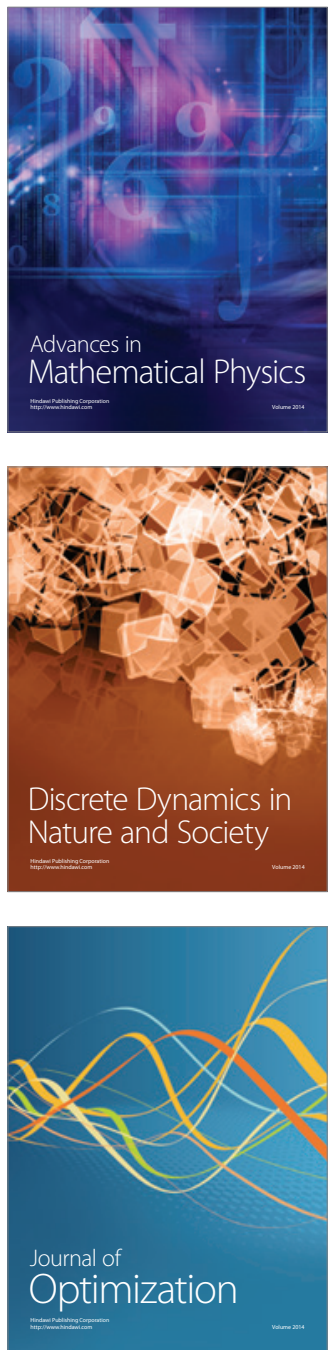